\title{
CARACTERIZACIÓN DE LEPIDÓPTEROS DIURNOS EN DOS SECTORES DEL SANTUARIO DE FLORA Y FAUNA LOS FLAMENCOS (SAN LORENZO DE CAMARONES, LA GUAJIRA)*
}

\author{
Georgina Paulina Moreno-M. ${ }^{1}$, Julio César Acuña-Vargas
}

\section{Resumen}

Con el fin de enriquecer el conocimiento sobre la biota del departamento de La Guajira se realizó el presente estudio, dirigido al conocimiento de la composición de lepidópteros diurnos presentes en dos sectores del área natural protegida y administrada por el Sistema de Parques Nacionales Naturales de Colombia, Santuario de Fauna y Flora Los Flamencos, La Guajira. La caracterización de lepidópteros diurnos se dirigió a individuos adultos, utilizando tres técnicas para su monitoreo (conteo visual sobre transecto lineal al azar sobre diferentes tipos de coberturas, trampas Van Someren-Rydon y recorridos libres con nasa para captura de mariposas). Se registran 1126 individuos del orden Lepidoptera, distribuidos en 3 familias, 9 subfamilias y 24 especies. El conocimiento de la diversidad biológica asociada a fragmentos de bosque seco tropical en La Guajira es necesario para la toma de decisiones sobre el uso y manejo de los recursos.

Palabras clave: Lepidoptera, santuario de vida salvaje, bosque seco tropical.

\section{CHARACTERIZATION OF DIURNAL LEPIDOPTERA IN TWO LOCATIONS IN SANTUARIO DE FLORA Y FAUNA LOS FLAMENCOS (SAN LORENZO DE CAMARONES, LA GUAJIRA)}

\begin{abstract}
In order to enrich the knowledge of the biota of the department of La Guajira this study led to the knowledge of the composition of diurnal Lepidoptera present in two sectors of the protected area and managed by the System of National Parks of Colombia, "Santuario de Fauna y Flora Los Flamencos", La Guajira. Characterization of diurnal Lepidoptera went to adult individuals, using three techniques for monitoring (visual count on transect at random from different types of coverage, traps Van Someren-Rydon and free tours with the butterfly net). 1126 individuals of the order Lepidoptera, divided into 3 families are recorded, 9 subfamilies, 24 species. Knowledge of biological diversity associated with fragments of tropical dry forest in La Guajira is necessary for decision-making on the use and management of natural resources, and even more in a biological group as Lepidoptera, which can be used as an indicator of disturbance due to their physiological characteristics and habitat specificity.
\end{abstract}

Key words: Lepidoptera, wildlife sanctuary, tropical dry forest.

\footnotetext{
*FR: 17-XI-2014. FA: .20-III-2015

${ }^{1}$ Biólogo, Grupo de Investigación en Ecología, Biodiversidad en Ecosistemas Tropicales (EBET), Universidad de La Guajira. Riohacha, Colombia. E-mail: gpmoreno@uniguajira.edu.co

${ }^{2}$ Biólogo, M.Sc(C) Ciencias Ambientales, Grupo de Investigación en Ecología, Biodiversidad en Ecosistemas Tropicales (EBET), Universidad de La Guajira.Riohacha, Colombia. E-mail: jcacuna@uniguajira.edu.co
} 


\section{INTRODUCCIÓN}

El Bosque seco tropical (Bs-t) en Colombia en 2008 era de aproximadamente 8’882.854 ha, hoy día resultado de la modelación climática, información cartográfica y de expertos, se estima que su cobertura es de 720 mil ha (PIZANO \& GARCÍA, 2014), de las cuales el Bs-t en la región Caribe presenta los remanentes más grandes y en mejor estado de conservación (55\% bosque natural) distribuido entre los 0-650 msnm (PIZANO \& GARCÍA, 2014), es considerado el ecosistema más amenazado en Colombia y del cual se posee menor conocimiento (PIZANO \& GARCÍA, 2014).

Cada fragmento de Bs-t es un eslabón importante para el conocimiento y conservación de este ecosistema, aunque la realidad actual es que los estudios sobre la historia, estado y desarrollo de la biota en este son escasos (IiAvHet al., 1998;PIZANO \& GARCÍA, 2014), presentándose la necesidad de generar conocimiento base sobre los diferentes grupos biológicos, su función y dinámica espacio-temporal en cada fragmento, que permitan el desarrollo de estrategias para su conservación. Es necesario identificar grupos biológicos que faciliten el conocimiento del estado del hábitat, que permitan identificar los cambios en la estructura y función del ecosistema.

Un hábitat adecuado es la necesidad primaria universalmente citada para la conservación, y hábitats inadecuados son las causas más citadas y omnipresentes de pérdidas y descensos (NEW, 2013). La pérdida y degradación de hábitat es la mayor amenaza para los lepidópteros, como para la mayoría de otros grupos taxonómicos (NEW, 2013); las poblaciones pueden disminuir rápidamente, debido a los cambios de hábitat o respuesta a la combinación de factores ambientales y genéticos, relacionados con las condiciones de hábitat favorables y la adaptación de estas a los mismos (BROWN,1984; NEW, 2013). Existen variados ejemplos de mariposas europeas, que se han visto diezmadas en las poblaciones (Chazara briseis) o desaparecidas (Maculinea arion), respuestas al desconocimiento de su historia natural, casos que se pudieron evitar y de los cuales se conocieron las causas después de sucedidos (NEW, 2013); lo cual demuestra que existe la necesidad de estudios dirigidos al conocimiento de la historia natural de las especies para la comprensión de su rol en los ecosistemas y sus necesidades biológicas, para identificar su hábitat adecuado.

Los lepidópteros son uno de los grupos de invertebrados con mayor diversidad en el Neotrópico y al igual que otro orden de insectos presentan mayor abundancia en la naturaleza (WOLFF, 2006). Estos constituyen una fracción de la biomasa animal de los ecosistemas; son elementos importantes en la dieta de muchos animales y asimismo cumplen funciones importantes como reguladores de las poblaciones vegetales (HOOK, 2009;NEW, 2013), además de desempeñar actividades de polinización de manera indirecta, liberan el néctar de una flor para trasladarlo a otras flores, favoreciendo la fecundación cruzada entre las especies vegetales (WINFREE et al., 2011).

Los lepidópteros son un grupo biológico que se sugiere pueden anunciar los cambios presentes en el clima local y global (KREMEN, 1992), son sensibles a los cambios presentes en el hábitat, que modifican las condiciones ambientales de los mismos, como la temperatura local y la humedad, además de la luminosidad entre otros (KREMEN, 1992; BROWN \& FREITAS, 2000; MONTERO-A. et al., 2009; 
ZAMBRANO-GONZÁLEZ \& ORTIZ-ORDÓÑEZ, 2009);su abundancia, conocimiento taxonómico y la distribución específica, respuesta a las condiciones ambientales de un determinado ecosistema, periodo, estación climática, nivel altitudinal, permiten utilizar este grupo como indicador del tipo y estado de hábitat (ANDRADE-C., 1998; WILSON et al., 2005; MONTERO-A. et al., 2009; ZAMBRANO-GONZÁLEZ \& ORTIZ-ORDÓÑEZ, 2009).

En búsqueda de enriquecer los registros biológicos dentro de los Bs-t se llevó a cabo el primer informe sobre la composición del orden Lepidoptera (diurna) en dos sectores del Santuario de Fauna y Flora Los Flamencos, con el fin de generar la información base para estudios futuros que permitan la toma de decisiones en la región y de manera especial en el área de estudio, la cual está dirigida a la conservación y protección de la biota.

\section{MATERIAL Y MÉTODOS}

\section{Área de estudio}

El Santuario de Flora y Fauna (SFF) Los Flamencos (11 $24^{\prime}$ N- $73^{\circ} 07^{\prime}$ O), es un área protegida natural ubicada en la costa Caribe en el norte de Colombia, con una extensión aproximada de 7682 ha, ubicadas en el corregimiento de San Lorenzo de Camarones, municipio de Riohacha, en el departamento de La Guajira; la altitud se distribuye entre los 0 a $5 \mathrm{msnm}$ con una temperatura promedio anual de $28-30^{\circ} \mathrm{C}$. El SFF Los Flamencos contiene cuatro lagunas costeras: laguna Navío quebrado, laguna Grande, laguna Manzanillo, laguna el Chentico, que presentan diferentes tipos de coberturas vegetales: manglar, bosque seco tropical, bosque ripario y matorrales subxerofíticos.

\section{Sectores de estudio}

El presente estudio se realizó en la región sur de la laguna Navío quebrado de San Lorenzo de Camarones, zona habitada por la comunidad indígena Wayuu y comunidades afrodescendientes, en esta zona se seleccionaron dos sectores de muestreo: el sector Pitilla $\left(11^{\circ} 23^{\prime} 23^{\prime \prime} \mathrm{N}-73^{\circ} 05^{\prime} 45^{\prime \prime} \mathrm{W}\right)$ y el sector Tococo $\left(11^{\circ} 22^{\prime} 58^{\prime \prime} \mathrm{N}\right.$ - 7305’22”W) (Figura 1).

Las lluvias marcan cambios en la estructura de las formaciones florísticas, observando aumento y disminución de las coberturas vegetales en relación al aumento y disminución de las precipitaciones; el sector Tococo muestra alta sensibilidad a la disminución de las precipitaciones, exhibiendo una apariencia más árida en comparación al sector Pitilla, principalmente en el periodo seco. 


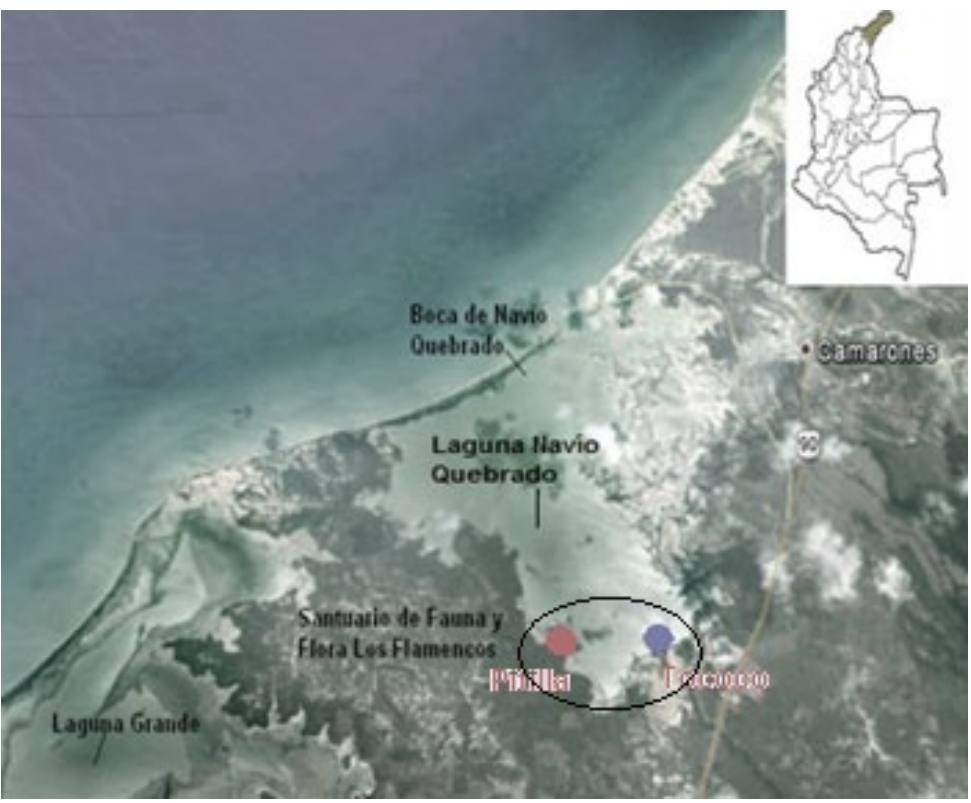

Figura 1. Mapa de ubicación de los sectores Pitilla y Tococo en el Santuario de Fauna y Flora Los Flamencos.

Las especies más representativas de la flora en los sectores de estudio son el Caranganito (Senna atomaria), el Corioto (Pithecellobium subglobosum), el Algarrobo (Ceratonia siliqua), el Barquito morado (Conmelina diffusa), especies que conservaron su follaje y presentan floraciones en el periodo seco; en menor abundancia se resalta la presencia de Cardón iguaraya (Stenocereus griseus), Cardón galluzo (Subpilocereus repandus), Pitahaya (Hylocereus undatus), Pichihuel (Melocactus curvispinus), Guamacho (Pereskia guamacho), Tuna (Opuntia caracasana), Túatúa (Jatropha gossypiifolia), Yuca guajira (Manihot carthaginensis),Volador (Ruprechtia ramiflora), Algondocillo (Bastardia viscosa), Babosa (Sida ciliaris), Barba e' sapo (Cleome spinosa), Coca de monte (Erythroxylum novogranatense), Mayita (Bromelia chrysantha),Batatilla morada (Ipomea carnea), Perrito o abrojo (Tribulus cistoides), Batatilla blanca (Ipomoea aegyptia), Bejuco cadena (Bauhinia splendens), Cinco llagas (Passiflora foetida), Escobilla (Melochia), Escobilla morada (Melochia tomentosa), Bejuco pega hueso (Serjania mexicana), Trupillo (Prosopis juliflora), Dividivi (Caesalpinia coriaria), Guayacán de bola (Bulnesia arborea), Manca mula (Bonellia rutescens),Leucaena (Leucaena leucocephala), Jobito o Caujaro (Cordia dentata), Mangle piñuelo o botoncillo (Conocarpus erectus), Huevo e' burro (Capparis pulcherrima), Olivo macho (Quadrella odoratissima), Aceituno (Vitex cymosa), Ceiba tolúa (Bombacopsis quinata), Bananito salado (Batis marítima); el perfil vegetal se distribuye entre los 0,35-10 m de altura.

Se resalta la condición de la cobertura vegetal del sector Pitilla, el cual presentó mejor condición y menor grado de perturbación en relación al sector Tococo. 


\section{MÉTODOS}

El presente estudio se realizó en el periodo comprendido entre los meses de mayo y octubre de2013, se realizaron 22 visitas a los sectores de estudio, dos días por localidad, con un esfuerzo de captura de 10 horas día, en el horario comprendido entre las 07:00-16:00 horas, con el fin de aprovechar la mayor actividad de las especies e incluir el mayor periodo de muestreo descrito en diferentes estudios (RAMÍREZ et al.,2007;MONTERO-A. et al., 2009;ZAMBRANO-GONZÁLEZ \& ORTIZORDÓÑEZ, 2009;PRINCE-CHACÓN et al., 2011), para un total de 220 horas hombre por localidad y un total de 440 horas para el estudio.

Se implementaron tres técnicas para el muestreo de lepidópteros diurnos:

Conteo visual sobre transecto lineal al azar sobre diferentes tipos de cobertura, técnica en la que se realizaron recorridos sobre transectos trazados al azar registrando la presencia, lugar y actividad de las especies.

Trampas Van Someren-Rydon,se utilizaron 12 trampas por localidad, separadas a una distancia de $10 \mathrm{~m}$ y ubicadas a una altura de $2 \mathrm{~m}$ cubriendo un área de $100 \mathrm{~m}$ por muestreo, se utilizaron frutas fermentadas y heces como sebos atrayentes, los cuales fueron cambiados según la necesidad, se monitorearon cada hora desde las 08:00 hasta las 15:00 obteniendo un esfuerzo de captura de 8 horas/día por trampa.

Recorrido libre y captura con redes para mariposa, técnica para abarcar mayor área y no limitar la búsqueda a transectos preestablecidos, coincide con el patrulleo mencionado por ANDRADE-C. (2007) citado en MONTERO-A. et al. (2009).

Se realizaron colectas manuales, dos ejemplares por especie, los cuales fueron sacrificados siguiendo los protocolos estandarizados para este grupo, y fueron transportadas en sobres de papel mantequilla, para posteriormente ser determinados (VILLAREAL et al., 2004; NEW, 2013).Los ejemplares fueron colectados, montados y rotulados siguiendo lo establecido en SAMWAYS et al. (2010); se depositaron en la colección de referencia de la Universidad de la Guajira, y se determinaron por medio de las especificaciones establecidas enguías de campo y claves dicotómicas (ANDRADE-C., 1990; VÉLEZ \& SALAZAR, 1991;ANDRADE-C. et al.,2007;MURILLO, 2008).

\section{ANÁLISIS DE RESULTADOS}

Las observaciones de especies en campo se organizaron en matrices de datos para su análisis, se organizó una lista de especies donde se registra lugar, fecha, hora, actividad de los ejemplares observados.

Por medio de curvas de acumulación de especies utilizando el programa StimateS 9.1.0 (COLWELL, 2013), se realiza el análisis de la riqueza observada frente a la riqueza estimada, utilizando los estimadores no paramétrico Jackknife de primer orden y Jackknife de segundo orden, que tienen en cuenta las especies que aparecen una sola vez (Uniques) o dos veces en la muestra (Duplicates) y por su uso recomendado en registros de presencia-ausencia (VILLAREAL et al.,2004), además se realizó un análisis de complementariedad de la diversidad de lepidópteros diurnos 
entre sectores, siguiendo lo descrito por COLWELL \& CODDINGTON (1994) citado en MORENO (2001).

\section{RESULTADOS}

Se registran 1126 individuos pertenecientes al orden Lepidoptera diurno presente en el SFF Los Flamencos, distribuido en tres de las seis familias registradas para el Neotrópico, 9 subfamilias, 20 géneros, 24 especies, 14 subespecies, siendo la familia Nymphalidae la que presenta mayor número de especies (16) que representan el $66,6 \%$ de los registros obtenidos (Tabla 1 ).

Tabla 1. Listado general de lepidópteros diurnos presentes en los sectores Pitilla y Tococo, SFF Los Flamencos.

\begin{tabular}{|c|c|c|c|c|c|}
\hline F amilia & Subfamilia & Género & Especie & Pitilla & Tococo \\
\hline \multirow[t]{16}{*}{ Nymphalidae } & Biblidinae & Marpesia & Marpesia petreus (Cramer, 1776) & $\mathrm{X}$ & $\mathrm{X}$ \\
\hline & & Hamadryas & Hamadryas februa ferentina (Godart, 1824) & $\mathrm{x}$ & $\mathrm{X}$ \\
\hline & & & Hamadryas feronia farinulenta (Fruhstorfer, 1916) & $\mathrm{x}$ & $\mathrm{x}$ \\
\hline & & Myscelia & Myscelia cyaniris cyaniris (Doubled ay, 1849) & $\mathrm{x}$ & $\mathrm{X}$ \\
\hline & Charaxinae & Fountainea & Fountainea glycerium glycerium (Doubleday, 1849) & $x$ & $\mathrm{x}$ \\
\hline & & Hypna & Hypna dytemnestra rufescens (Butler, 1866) & $\mathrm{x}$ & $\mathrm{X}$ \\
\hline & & Cymatogramma & Cymatogramma pithyusa pithy usa (Felder, 1869) & & $\mathrm{X}$ \\
\hline & Danainae & Danaus & Danaus gilippus thersippus (Bates, 1863) & $\mathrm{X}$ & \\
\hline & & & Danaus erippus (Cramer, 1775) & $\mathrm{x}$ & \\
\hline & & & Danaus eresimus (Cramer, 1777) & $\mathrm{x}$ & $\mathrm{x}$ \\
\hline & Heliconiinae & Agraulis & Agraulis vanillae (Linnaeus, 1758) & $\mathrm{x}$ & $\mathrm{x}$ \\
\hline & & Euptoieta & Euptoieta hegesia hoffmanni (Comstock, 1944) & $\mathrm{x}$ & \\
\hline & & Heliconius & Heliconius erato hydara (Hewitson, 1867) & $\mathrm{X}$ & $\mathrm{X}$ \\
\hline & Nymphalinae & Anartia & Anartia jatrophaes (Linnaeus, 1763) & $\mathrm{X}$ & \\
\hline & & Junonia & Junonia evarete (Cramer, 1779) & $\mathrm{x}$ & $\mathrm{X}$ \\
\hline & Satyrinae & Taygetis & Taygetis laches (Fabricius, 1793) & $\mathrm{x}$ & \\
\hline Papilionidae & Papilioninae & Heraclides & Heradides homothoas (Rothschild \& Jordan, 1906) & & $\mathrm{x}$ \\
\hline \multirow[t]{8}{*}{ Pieridae } & Coliadinae & Anteos & Anteos maerula (Fabricius, 1775) & $\mathrm{x}$ & \\
\hline & & Aphrissa & Aphrissa statira statira (Cramer, 1777) & $\mathrm{X}$ & \\
\hline & & Phoebis & Phoebis argante (Fabricius, 1775) & $\mathrm{x}$ & $\mathrm{X}$ \\
\hline & & & Phoebis sennae marcellina (Cramer, 1777) & $\mathrm{X}$ & $\mathrm{X}$ \\
\hline & & & Phoebis sennae sennae (Linnaeus, 1758) & $\mathrm{x}$ & $\mathrm{X}$ \\
\hline & & Pyrisitia & Pyrisitia limbia & $\mathrm{x}$ & $\mathrm{x}$ \\
\hline & Pierinae & Appias & Appias drusilla (Cramer, 1777) & $\mathrm{x}$ & \\
\hline & & Ascia & Ascia monuste (Linnaeus, 1764) & $\mathrm{x}$ & \\
\hline
\end{tabular}

En relación a las subfamilias, el mayor número de especies (6) lo representa la subfamilia Coliadinae de la familia Pieridae que representa el 25\% de la riqueza total para el presente estudio, seguido de la subfamilia Biblidinae(4) de la familia Nymphalidae representando el16,66\%; Charaxinae, Danainae, Heliconiinae de la familia Nymphalidae (3) 12,5\% cada una, representando el 37,5\% del total de especies registradas; Nymphalinae de la familia Nymphalidae y Pierinae de la familia Pieridae (2) representan cada una el 8,33\% y el 16,66\%; Satyrinae de la familia Nymphalidae y Papilioninae de la familia Papilionidae (1) cada una representan el 4,16\% y el 8,33\% de las especies registradas para el presente estudio (Tabla 1).

El sector que presenta el mayor número de familias (3) es Tococo, los dos sectores presentan el mismo número de subfamilias (8), mientras que la mayor representación de géneros (18) y especies (22) se encuentra en el sector Pitilla (Tabla 1). 


\section{Sector Pitilla}

El sector Pitilla registra 735 individuos, 65\% del total de individuos observados, los cuales se encuentran distribuidos en dos familias (Pieridae 52\%, Nymphalidae 48\%), 8 subfamilias, 18 géneros, 22 especies de mariposa diurnas; siendo la familia Nymphalidae la que presenta mayor número de especies (15). Los mayores registros de riqueza de especies se presentaron en los meses de junio (13), septiembre (13) y octubre (18) respectivamente.

En relación a las subfamilias, el mayor número de especies (4) lo presenta Biblidinae de la familia Nymphalidae representando el 18,18\% de la riqueza obtenida para este sector; seguido por las subfamilias Danainae y Heliconiinae de la familia Nymphalidae y Coliadinae de la familia Pieridae, con tres especies cada una, que representan el 40,9\% de las especies registradas para el sector Pitilla (Figura 2).

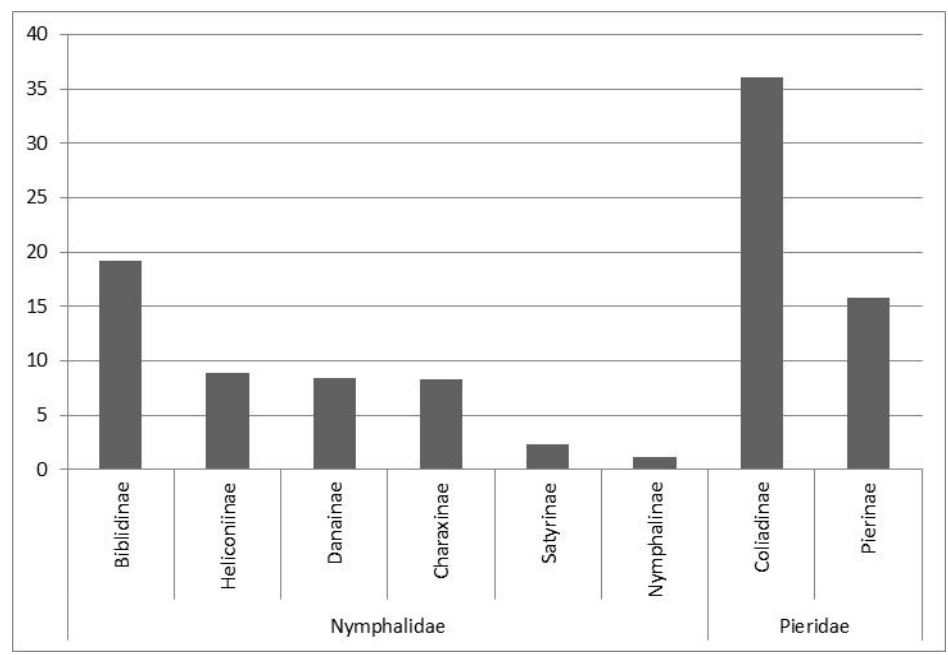

Figura 2. Riqueza de especies en cada familia y subfamilia de lepidópteros diurnos registrados en el sector Pitilla, SFF Los Flamencos.

Se resaltan como especies únicas (9) para el sector Pitilla, las especies Taygetis laches laches, Danaus erippus, Danaus gilippus,Euptoieta hegesia hoffmanni y Anartia jatrophae, pertenecientes a la familia Nymphalidae; Anteos maerula,Aphrissa statira statira, Appias drusilla y Ascia monuste, pertenecientes a la familia Pieridae.

La especie más abundante es Ascia monuste con 97 individuos que representan el $13,19 \%$ de la abundancia, seguido por las especies Hamadryas feronia farinulenta (77 individuos), Phoebis sennae marcellina (74 individuos) y Anteos maerula (68 individuos); las cuatro especies representan el 42,99\% de la abundancia total para este sector. La familia que presentó mayor abundancia es la familia Pieridae, la cual representa el 51,83\% de la abundancia de lepidópteros diurnos registrados; las subfamilias más representativas son: Coliadinae (36,05\%), Biblidinae $(19,18 \%)$ y Pierinae (15,78\%) (Figura 3). 


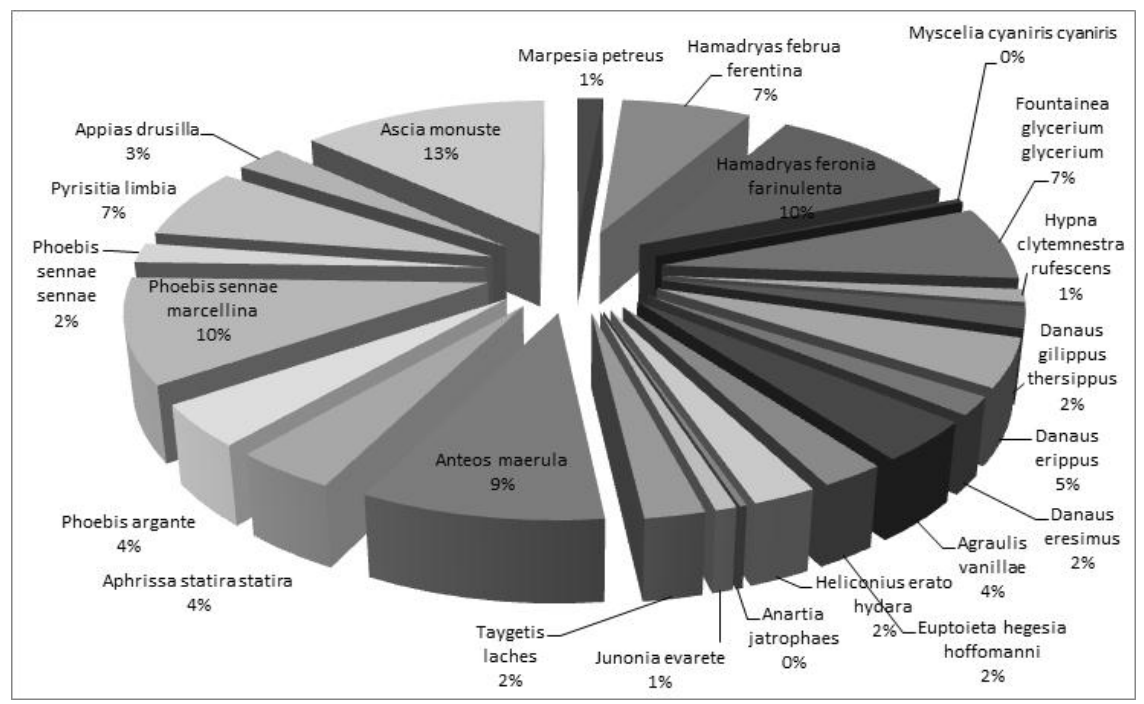

Figura 3. Distribución porcentual de registro de especies de lepidópteros diurnos en el sector Pitilla, SFF Los Flamencos.

El estimador no paramétrico Jackknife de primer orden muestra que las especies encontradas tienen una representatividad del 93,23\% de las especies esperadas, relación similar presenta el estimador de Jackknife de segundo orden con un valor mayor de completitud, presentado una representatividad del 99,13\% de las especies esperadas para este sector (Figura 4).

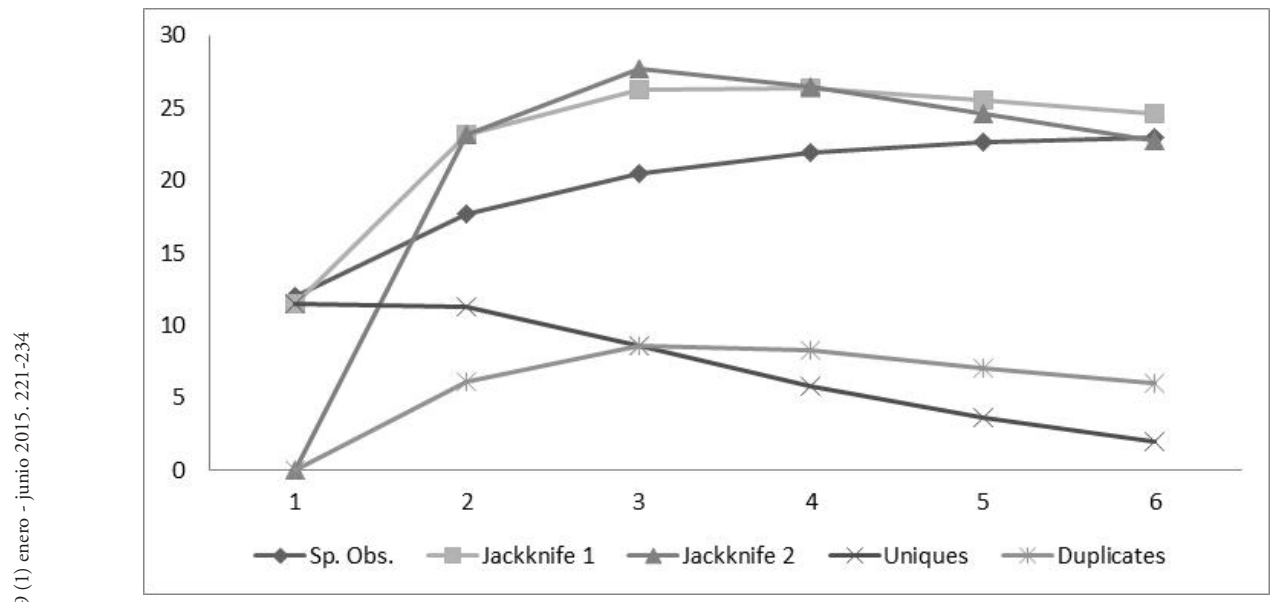

Figura 4. Curvas de acumulación de especies para el sector Pitilla, SFF Los Flamencos, utilizando los estimadores Jackknife de primer y segundo orden y las especies únicas y duplicadas. 


\section{Sector Tococo}

El sector Tococo registra 391 individuos, 35\% del total de lepidópteros observados, los cuales se encuentran distribuidos en tres familias (Nymphalidae 64,7\%, Pieridae 29,4\%, Papilionidae 5,9\%), 8 subfamilias, 13 géneros y 15 especies de mariposa diurnas, siendo la familia Nymphalidae la mejor representada en número de especies (11).Los mayores registros de riqueza de especies se presentaron en los meses de julio (5), septiembre (6) y octubre (16) respectivamente.

En relación a las subfamilias, el mayor número de subfamilias (8) para este estudio lo presenta este sector; el mayor número de especies (4) lo presenta Biblidinae de la familia Nymphalidae representando el 26,66\% de la riqueza obtenida para este sector; seguido por las subfamilias Characinae de la familia Nymphalidae y Coliadinae de la familia Pieridae, con tres especies cada una, que representan el 40\%; Heliconiinae de la familia Nymphalidae, con dos especies, representa el 13,33\%; Danainae y Nymphalinae de la familia Nymphalidae y Papilioninae de la familia Papilionidae con una especie, representando el 20\% de las especies registradas para el sector Tococo (Figura5).

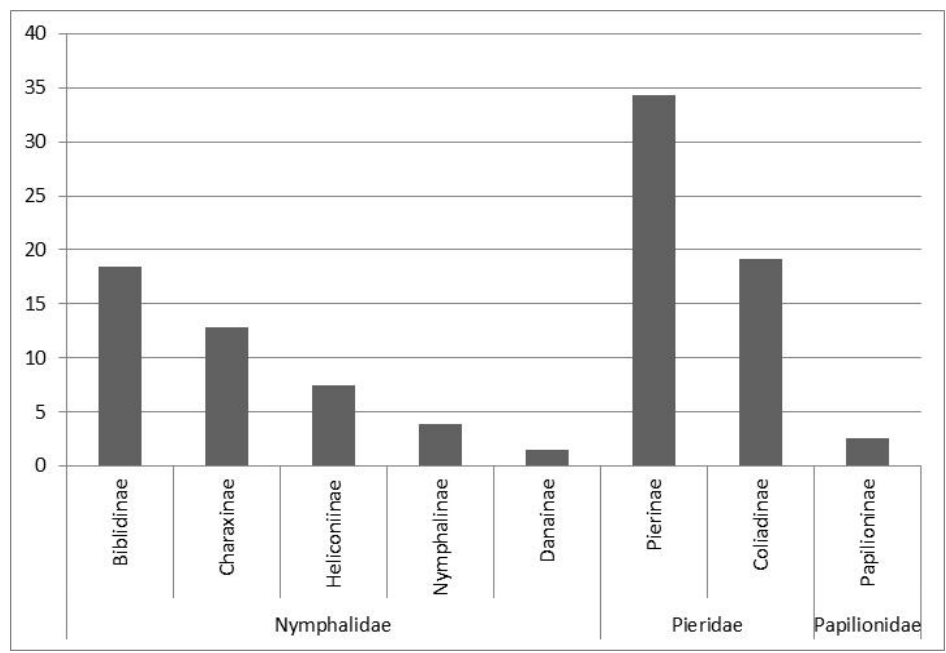

Figura 5. Riqueza de especies en cada familia y subfamilia de lepidópteros diurnos registrados en el sector Tococo, SFF Los Flamencos.

Se resaltan como especies únicas para el sector Tococo: Cymatogramma pithyusa pithyusa perteneciente a la familia Nymphalidae y el único registro de la familia Papilionidae, subfamilia Papilioninae, con la especie Heraclides homothoas, las cuales representan el 13,33\% de las especies observadas para este sector.

La especie más abundante es Ascia monuste con 88 individuos que representan el 22,5\% de la abundancia, seguido por las especies Appias drusilla (77 individuos), Hamadryas februa ferentina (42 individuos) y Cymatogramma pithyusa pithyusa (25 individuos); las cuatro especies representan el 51,4\% de la abundancia total 
para este sector. La familia que presentó mayor abundanciaes la familia Pieridae, la cual representa el 53,45\% de la abundancia de lepidópteros diurnos registrados; las subfamilias más representativa son: Pierinae $(34,27 \%)$, Coliadinae $(19,18 \%)$ y Biblidinae (18,41\%) (Figura 6).

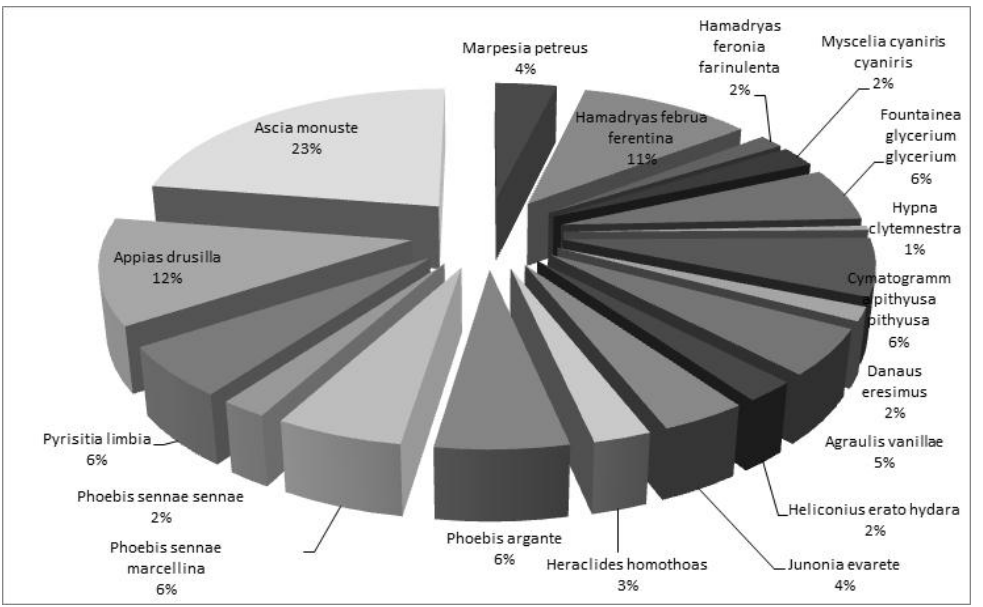

Figura 6. Distribución porcentual de registro de especies de lepidópteros diurnos en el sector Tococo, SFF Los Flamencos.

El estimador no paramétrico Jackknife de primer orden, muestra que las especies encontradas tienen una representatividad del 75,53\% de las especies esperadas, relación similar presenta el estimador de Jackknife de segundo orden, con una representatividad del $71,14 \%$ de las especies esperadas; los resultados obtenidos muestran que para este sector es posible encontrar más especies de lepidópteros diurnos (Figura 7).

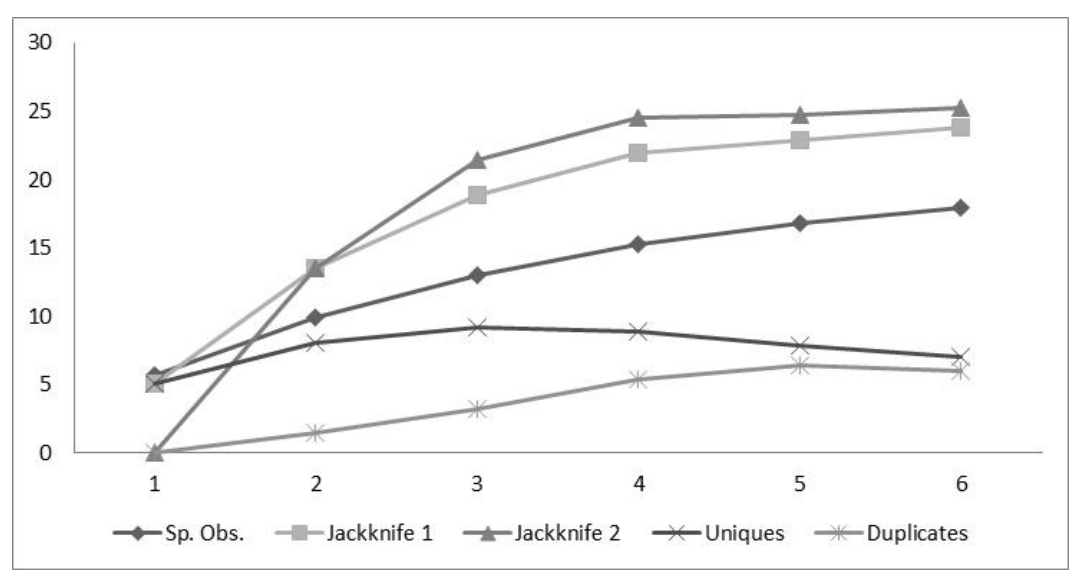

Figura 7. Curvas de acumulación de especies para el sector Tococo, SFF Los Flamencos, utilizando los estimadores Jackknife de primer y segundo orden y las especies únicas y duplicadas. 
Teniendo en cuenta el número de especies encontradas y exclusivas en los sectores Pitilla y Tococo, la diversidad de lepidópteros diurnos es complementaria en su composición de especies en un 41\% (Tabla 2).

Tabla 2. Índice de complementariedad para los sectores Pitilla y Tococo, SFF Los Flamencos.

\begin{tabular}{lr}
\hline \multicolumn{2}{c}{ COMPLEMENTARIEDAD } \\
\hline Número de especies Sector Pitilla (A) & 22 \\
Número de especies Sector Tococo (B) & 16 \\
Número de especies comunes (C) & 14 \\
Número especies exclusivas Pitilla & 7 \\
Número especies exclusivas Tococo & 2 \\
$\mathrm{SAB}=\mathrm{A}+\mathrm{B}-\mathrm{C}$ & 24 \\
$\mathrm{UAB}=\mathrm{A}+\mathrm{B}-2 \mathrm{C}$ & 10 \\
$\mathrm{CAB}=\mathrm{UAB} / \mathrm{SAB}$ & 0,41 \\
\hline
\end{tabular}

\section{DISCUSIÓN}

En el SFF Los Flamencos, sectores Pitilla y Tococo, se encuentran representadas tres de las seis familias de mariposas del Neotrópico (MONTERO-A. et al., 2009); la familia Nymphalidae aporta el mayor número de subfamilias, géneros y especies, lo cual es similar a lo registrado para otros estudios en Colombiay puede ser respuesta a la alta riqueza y diversidad de hábitos alimenticios del grupo (WOLFF, 2006; CAMPOS-SALAZAR et al., 2011; PRINCE-CHACÓN et al., 2011).

La familia Papilionidae fue la que presentó menor riqueza, lo que pude ser explicado según lo expuesto por otros autores a la ausencia o escasez de plantas hospederas Mimosaseae y Aristolocheaceae (PRINCE-CHACÓN et al., 2011).

Se resalta la presencia de la especie Taygetis laches solo para el sector Pitilla, especie asociada a áreas poco alteradas o con acceso fácil a la oferta trófica, de igual manera se observa la especie Heliconius erato en los dos sectores, especie asociada a áreas intervenidas (ANDRADE-C. et al., 2007; PRINCE-CHACÓN et al., 2011); las dos especies presentaron abundancia total similar (17 y 18 individuos, respectivamente) en el sector Pitilla, con diferente distribución espacio-temporal; la especie Taygetis laches se observó solo en el mes de octubre y Heliconius erato en los meses de junio, julio y octubre, este último presentó la mayor abundancia (12 individuos). Al revisar las notas de CAMPOS-SALAZAR et al. (2011) sobre Taygetis laches y su baja colecta, puede relacionarse su presencia a la condición de la cobertura vegetal, menos intervenida en el sector Pitilla en relación al sector Tococo el cual no presentó registros de la especie; la presencia de las especies por sí sola no explica la condición del sitio de estudio.

Las especies que registran mayor abundancia en el sector Pitilla, Hamadryas feronia, Anteos maerula, Phoebis sennae marcellina y Ascia monuste, tienen en común ser especies asociadas a zonas abiertas, perturbadas, nectarívoras de variadas especies de flores; mientras que las especies más abundantes presentes en el sector Tococo, 
Hamadryas februa, Appias drusilla y Ascia monuste, se resaltan por tener en común ser nectarívoras de variadas especies y habitar zonas perturbadas como bordes de bosque, jardines y áreas cultivadas con árboles.

Las especies Hypna clytemnestra rufescens y Cymatogramma pithyusa pithyusa, se encontraron relacionadas a zonas más sombrías y con mayor estrato.

Las especies de mariposas registradas en el estudio guardaron relación con la vegetación presente en el área de estudio; Agraulis vanillae, Ascia monuste, Appias drusilla, Heraclides homothoas, Pyrisitia limbia entre otras especies se encontraron libando entre flores de árboles, plantas y arbustos fértiles participando de la polinización de la vegetación como Dividivi, Guamacho, Olivo macho, Agua amarga, Aceituno, Ceiba tolúa, Guayacán de bola, Algarrobo dulce y en otro tipo de vegetación en tiempo de floración, lo cual hace a este grupo de gran importancia para la evaluación y recuperación de los ecosistemas (CONSTANTINO,1997).

Se observaron especies de la subfamilia Coliadinae asociadas a las zonas de mayor intervención en los dos sectores de estudio, lo cual se relacionó a lo encontrado por LAWTON et al. (1998) y RICKETTS et al. (2002) en sus investigaciones, donde identifican a las especies de la subfamilia Coliadinae como tolerantes a paisajes intervenidos y de alto deterioro natural o antropogénicos.

Representantes de las subfamilias Biblidinae, Charaxinae y Nymphalinae se observaron en lugares con cobertura vegetal medianamente intervenida en los dos sectores de estudio, lo cual es soportado en lo manifestado por OROZCO et al. (2009) quienes referencian estas subfamilias en su trabajo desarrollado en áreas de bosque seco tropical presente en el Occidente Antioqueño, asociando a las especies de estas familias relacionadas a paisajes poco intervenidos.

La especie Danaus eresimus se registró la mayoría de las veces cerca de la laguna Navío quebrado y en lugares húmedos, lo cual puede ser explicado por RAMíREZ et al. (2007) quienes encontraron que esta especie se caracteriza por habitar zonas húmedas, inundables, como los sistemas de humedales y lagunas originados por la influencia de ríos.

Al igual que en otros estudios para la región Caribe de Colombia se registra la presencia de Hypna clytemnestra rufescens, la cual restringe su distribución a la costa Caribe (MONTERO-A. et al., 2009).

Aunque se menciona la relación de algunos grupos y especies con características propias, fácilmente identificables, que permitirían la presunción de tendencias base para ser indicadores de las condiciones de hábitat o de ecosistemas, se manifiesta que el uso de algún grupo de mariposas como indicador en los ecosistemas de cualquier sitio o de la utilización de estas para medir la calidad de un hábitat, debe hacerse con precaución, debido a que grupos ecológicos diferentes pueden tener requerimientos ecológicos distintos (OROZCO et al., 2009) y es algo que se debe tener en cuenta en investigaciones futuras, dado que no es un objetivo directo de la presente investigación.

La distribución de la riqueza y abundancia de especies está relacionada a la combinación de ofertas ambientales presentes en el ecosistemas, variables físicas 
como temperatura, humedad, brillo solar, velocidad del viento y variables biológicas como relación planta hospedero (larvas), relación frutos y flores (adultos), entre otras, que influyen de manera directa e indirecta sobre la dinámica espaciotemporal de las mariposas diurnas como lo soportan diversos autores (BROWN, 1984; RAMÍREZ et al., 2007; MONTERO-A. et al., 2009; TOBAR \& IBRAHIM, 2010).

La necesidad de información en la región Caribe sobre la riqueza de especies, comportamiento y función de las mariposas asociadas a fragmentos de bosque seco tropical, son una realidad a asumir para lograr evaluar de manera rápida el estado de los fragmentos y priorizar su conservación (DEVRIES et al., 1999; MONTERO-A. et al., 2009; PRINCE-CHACÓN et al., 2011).

\section{CONCLUSIONES}

El Santuario de Fauna y Flora Los Flamencos registra para el presente estudio 24 especies distribuidas en seis familias de lepidópteros diurnos. Las especies que presentaron mayor abundancia en los dos sectores de muestreo están asociadas a diferentes grados de perturbación en el hábitat.

Los análisis de la riqueza de especies registrados muestran que existe una gran posibilidad de registrar más especies para cada sector de muestreo.

La dinámica espacio-temporal de las mariposas diurnas se encuentra relacionada con diversos factores entre los que sobresalen los cambios en los elementos del clima y las condiciones del hábitat, lo cual permite o favorece la diversidad. Elementos básicos del monitoreo de las poblaciones de este grupo como la riqueza y algunos aspectos ecológicos, constituyen una herramienta válida para evaluar en qué estados de conservación o alteración se encuentran las áreas en las cuales se distribuyen.

Este estudio es el primer acercamiento al conocimiento de los lepidópteros presentes en el SFF Los Flamencos y la base para estudios posteriores dirigidos al conocimiento de la riqueza y función de estos en esta área destinada a la conservación de la biota.

\section{AGRADECIMIENTOS}

Los autores presentan sus agradecimientos a quien permitió la realización del presente estudio y a todos los que colaboraron para culminarlo, en especial a Grescia Moreno Muñiz, Soleida PushainaIpuana y Mileidys Sierra Julio, por su invaluable apoyo y colaboración; al personal administrativo y operativo del Parque Nacional Natural, Santuario de Flora y Fauna Los Flamencos, en especial a Ilder Gómez Sierra y Robinson Galindo Tarazona; a la Universidad de La Guajira, Facultad de Ciencias Básicas y Aplicadas, Laboratorio de Ciencias Biológicas y las colecciones biológicas de la Universidad de La Guajira, en especial al personal del herbario y al grupo de investigación Ecología, Biodiversidad en Ecosistemas Tropicales (EBET). 


\section{BIBLIOGRAFÍA}

ANDRADE-C., M., 1990.-Clave para las familias y subfamilias de Lepidoptera: Rhopalocera de Colombia. Caldasia, 16: 197-200.

1998.- Utilización de las mariposas como bioindicadoras del tipo de hábitat y su biodiversidad en Colombia. Revista de la Academia Colombiana de Ciencias, 22(84): 407-421.

ANDRADE-C., M., CAMPOS, L., GONZÁLEZ, L.\& PULIDO-B., H.W., 2007.-Santa María Mariposas alas y color. Instituto de Ciencias Naturales, Universidad Nacional de Colombia, Bogotá D.C.

BROWN, J., 1984.- On the relationship between abundance and distribution of species. The American Naturalist, 124 (2): 255-279.

BROWN, K. \& FREITAS, A., 2002.-Atlantic Forest Butterflies: Indicators for Landscape Conservation. Biotropica, 32: 934-956.

COLWELL, R.K., 2013.-StimateS: Statistical estimation of species richness and shared species from samples. Version 9.

CAMPOS-SALAZAR, L., GÓMEZ, L. \& ANDRADE-C., M., 2011.- Mariposas (Lepidoptera: Hesperioidea Papilionoidea) de las áreas circundantes a las ciénagas del departamento de Córdoba. Revista de la Academia Colombiana de Ciencias, 35 (134): 45-60.

CONSTANTINO, L.M., 1997.-Lepidópteros Diurnos del Choco Biogeográfico: Diversidad, alternativas productivas sostenibles y estrategias de conservación.Memorias XXIV Congreso Sociedad Colombiana de Entomología.

DEVRIES, P., WALLAS, T. \& GREENEY, H., 1999.- Species diversity in spatial and temporal dimensions of fruitfeeding butterflies from two Ecuadorian rainforests. Biological Journal of the Linnean Society, 68: 333-353.

HOOK, P., 2009.-Mariposas: 42-101 (en) HOOK, P. Insectos de todo tipo. ParragonBooksLtd, Reino Unido.

INSTITUTO ALEXANDER VON HUMBOLDT, PROGRAMA DE INVENTARIO DE LA BIODIVERSIDAD \& GRUPO DE EXPLORACIONES Y MONITOREO AMBIENTAL GEMA., 1998.-El Bosque seco Tropical (Bs-t) en Colombia. Instituto Alexander von Humboldt, Villa de Leyva.

KREMEN, C., 1992.-Assessing the indicator properties of Species Assemblages for Natural Areas Monitoring. Ecological Applications, 2: 203-217.

LAWTON, J., BIGNELL, D., BOLTON, B., BLOEMERS, G., EGGLETON, P.,HAMMOND, P., HODDA, M., HOLT, R., LARSEN, T., MAWDSLEY, N., STORK, N., SIVASTRAVA, D.\& WATT, A., 1998.-Biodiversity inventories, indicator taxa and effects of habitat modification in tropical forest. Nature, 391(1): 72-76.

MONTERO-A., F., MORENO-P., M. \& GUTIÉRREZ-M., L., 2009.-Mariposas (Lepidoptera: Hesperioidea y Papilionoidea) asociadas a fragmentos de bosque seco tropical en el departamento del Atlántico, Colombia. Bol. Cient. Mus. Hist. Nat. U. de Caldas,13 (2): 157-173.

MORENO, C., 2001.-Métodos para medir la Biodiversidad. Vol. 1. M\&T-Manuales y Tesis SEA, Zaragoza.

MURILLO, H.L.R., 2008.- Clave dicotómica para la identificación de las familias de mariposas (Rhopalocera) pertenecientes a las superfamiliasPapilionoidea y Herperioidea. MES, 3(2): 6-11.

NEW, T.R., 2013.-Lepidoptera and conservation.Wiley Blackwell.

OROZCO, S., MURIEL.S.\& PALACIO, J., 2009.- Diversidad de Lepidópteros diurnos en un área de Bosque seco Tropical del occidente Antioqueño. Actualidades Biológicas,31 (90): 31- 41.

PIZANO, C. \& GARCÍA, H., 2014.-El Bosque seco tropical en Colombia. Instituto de Investigación de Recursos Biológicos Alexander von Humboldt, Bogotá, D.C.

PRINCE-CHACÓN, S., VARGAS-ZAPATA, M., SALAZAR-E., J. \& MARTÍNEZ-HERNÁNDEZ, N.,2011.- Mariposas Papilionoidea y Hesperioidea (Insecta: Lepidoptera) en dos fragmentos de bosque seco tropical en Corrales de San Luis, Atlántico, Colombia. Boletín de la Sociedad Entomológica Aragonesa, 48: 243-252.

RAMÍREZ, L., CHACÓN, P.\& CONSTANTINO, L., 2007.- Diversidad de mariposas diurnas (Lepidóptera: Papilionoidea y Hesperioidea) en Santiago de Cali, Valle del Cauca, Colombia. Revista Colombiana de Entomología, 33 (1): 54-63.

RICKETTS, T., DAILY, G. \& EHRLICH, P., 2002.- Does butterfly diversity predict moth diversity? Testing a popular indicator taxon at local scales.Biological Conservation, 10 (3): 361-370.

SAMWAYS, M., MCGEOCH, M.\& NEW, T., 2010.-Insect conservation: a handbook of approaches and methods. Oxford Universitypress, New York.

TOBAR, D. \& IBRAHIM, M., 2010.- ¿Las cercas vivas ayudan a la conservación de la diversidad de mariposas en paisajes agropecuarios? Biología Tropical, 58 (1): 447-463.

VÉLEZ, J. \& SALAZAR, J., 1991.-Mariposas de Colombia. Villegas Editores, Bogotá, D.C.

VILLAREAL, H., ÁlVAREZ, M., CÓRDOBA, S., ESCOBAR, F., FAGUA, G., GAST, F., MENDOZA, H., OSPINA, M.\& UMAÑA, A.M., 2004.-Manual de métodos para el desarrollo de inventarios de Biodiversidad. Programa de inventarios de Biodiversidad, Instituto de Investigación de Recursos Biológicos Alexander von Humboldt. Bogotá, D.C.

WILSON, R., GUTIÉRREZ, D., GUTIÉRREZ, J., MARTÍNEZ, G., AGUDO, R.\& MONTSERRAT, V., 2005.-Changes to the elevational limits and extent of species ranges associated with climates change.Ecology Letters, 8 (11): 1138-1146

WINFREE, R., BARTOMEUS, I.\& CARIVEAU, D.P., 2011.- Native pollinators in anthropogenic habitats. Annual Review of Ecology, Evolution and Systematics, 42: 1-22.

WOLFF, M., 2006.-Orden Lepidoptera: 332-365 (en)WOLFF, M. Insectos de Colombia, Guíabásica de familias. Multiempresos, Medellín.

ZAMBRANO-GONZÁLEZ, G. \& ORTIZ-ORDÓÑEZ, G., 2009.- Diversidad de lepidópteros diurnos en tres localidades del corredor biológico y multicultural Munchique-Pinche, Cauca, Colombia. Bol. Cient. Mus. Hist. Nat. U. de Caldas,13 (1): 241-224. 